Revista Mexicana de Economía y Finanzas Nueva Época, Vol. 13 No. 4, (2018), pp. 483-505

DOI: http://dx.doi.org/10.21919/remef.v13i4.334

\title{
What makes Input-Output Tables of Trade of Raw Material Goods Peculiar Networks? The World and Mexican Cases ${ }^{1}$
}

\author{
Katya Pérez-Guzmán \\ International Institute for Applied Systems Analysis, Austria \\ Isela-Elizabeth Téllez-León \\ International Institute for Applied Systems Analysis, Austria \\ Ali Kharrazi \\ The University of Tokyo, Japan \\ Brian Fath \\ Towson University, USA \\ Francisco Venegas-Martínez ${ }^{2}$ \\ Instituto Politécnico Nacional, Mexico \\ (Recepción: 23/febrero/2018, aceptado: 31/julio/2018)
}

\section{Abstract}

Objective: This paper examines several peculiarities of Input-Output Tables (IOTs) of trade of raw material goods when treated as networks. Methodology: two IOTs of trade of raw material goods (World and Mexico) are compared with a network with a scale free distribution and hierarchical organization (an email database) by using distinct centralities and statistics from graph theory. Results: IOTs are a very particular type of graph due to their idiosyncrasies, for which standard graph measures cannot provide satisfactory results, and which have to be adapted to give a fragmented picture of the whole network. Recommendations: analytical tools of network theory applied to IOTs substantially improve the understanding of trade of raw material goods, both at the national and the global level, which is useful in the design of trade policy. Limitations: this research does not include random walk centrality and regime switching from external shocks. Originality: this is a novel research that enlightens the particularities of the IOTs, seen as networks, for the Mexican case. Conclusions: this investigation finds important particularities of IOTs when compared with other networks.

JEL Classification: C40, C67, F10

Keywords: Network analysis, network topology, graph theory, input-output tables, extractivism, raw material trade

\section{¿Qué hace que las tablas insumo-producto del comercio de mercancías de materia prima sean redes peculiares? Los casos mundial y mexicano}

\section{Resumen}

Objetivo: se examinan varias peculiaridades de las tablas de input-output (IOT) del comercio de materias primas cuando se tratan como redes. Metodología: dos IOTs de comercio de materias primas (mundial y México) se comparan con una red con distribución de escala y organización jerárquica (una base de datos de correos electrónicos) utilizando distintas centralidades y estadísticas de la teoría de grafos. Resultados: las IOTs son un

\footnotetext{
${ }^{1}$ Special thanks are due to the referees for valuable and helpfull comments and suggestions.

${ }^{2}$ Corresponding author. Instituto Politécnico Nacional, Mexico. Email: fvenegas1111@yahoo.com.mx
} 
484 REMEF (The Mexican Journal of Economics and Finance)

What makes Input-Output Tables of Trade of Raw Material Goods Peculiar Networks? The World and Mexican Cases

\section{Resumen}

tipo de gráfico muy particular debido a su idiosincrasia, para las cuales las medidas de estándar de gráficas no proporcionan resultados satisfactorios, y que deben adaptarse para dar un retrato fragmentado de toda la red. Recomendaciones: las herramientas analíticas de redes aplicadas a las IOTs mejoran la comprensión del comercio de materias primas, a nivel nacional como mundial, lo cual es útil en el diseño de la política comercial. Limitaciones: no se incluye la centralidad de caminata aleatoria ni cambios de régimen por shocks externos. Originalidad: es una contribución novedosa que resalta particularidades de las IOTs, vistas como redes, para México. Conclusiones: se encuentran importantes particularidades de las IOTs al compararlas con otras redes.

Clasificación JEL: C40, C67, F10

Palabras clave: Análisis de redes, topología de red, teoría de grafos, tablas input-output, extractivismo, comercio de materias primas

\section{Introduction}

In the last decade, the analysis of an Input-Output Table (IOT) has been used in many novel applications for economic policy design due to their structured information, ease of access and manipulability, as well as the straight link with comparative national accounts. A further utility of an IOT is its relational nature, which allows for analytical inquiry using graph or network theories.

Many papers have focused on network analysis of IOTs. A subgroup of these papers attempts to characterize global trade by determining the topology, the most important nodes and communities, the trade partner preference, and the measurement of various interregional flows with hybrid databases. However, there are potential applications that require a clearer perspective of methodological issues related to adapting graph theory tools in IOT analysis. The main objective of the present paper is to state several methodological reflections about what makes IOTs different from other networks. In particular, this paper examines the global trade in raw material goods to show how tools of graph theory can adapted for network analysis.

The organization of the paper is as follows: the first section sets up the basic concepts regarding network analysis and IOTs, and presents a short literature review on IOT network analysis of trade; the second section provides the methodology use in the research; the third section provides a suitable network analysis of IOT through a straightforward comparison among three digraphs, one with properties common to complex networks, and other two of national and world IOTs, which enlightens the particularities of the IOTs; the last section concludes with a reflection of potential areas of research.

\section{Network analysis of IOTs}

Network theory studies graphs as a representation of relations among discrete objects. A network can be defined as a graph in which nodes (vertices) and links (edges or arrows) have attributes. Two nodes are connected if they share an edge between them through an interrelation of any type. This adjacent pair-wise exchange is the foundation for creating the network. In addition, the connections give rise to qualitative relations which are influenced by the entire web of interactions. This relation can be numerically ordered, with some nodes having higher ranks than others in terms of various measures. Network analysis concerns itself most commonly with two main characteristics: the structure or topology of the entire network (the arrangement of the nodes and links.) and the importance 
(attributes) of specific nodes given such topology. The structure of the entire network is studied by analyzing related subsets of nodes and edges in groups or paths, and concerns itself with various qualities, such as the patterns of connections between nodes, how flows permeate through the entire network, or how groups of nodes cluster with each other. An advantage of this approach is that it allows for a whole-scale systems analysis that the contribution of all nodes be simultaneously considered. A network can have a static topology or it can change in time (switching regimes) due to exogenous shocks.

Network theory is an emerging field of research useful to analyze, describe and explain financial and economic interrelations of agents. The use of methods from network theory has improved the understanding of systems in which social the interrelations of agents cannot be ignored. In this case, it is necessary to use a suitable framework to depict the composition of nodes, links and attributes; this is precisely what network theory does. Representing a problem as a graph may provide different points of view for finding solutions, and setting a problem as a graph may provide a simpler analysis of the possible solutions. Needless to say, network theory has applications in many disciplines including economics, finance and other social sciences; see, for instance, Jackson (2008). Applications of network theory include: systemic risk and stability in financial networks (Acemoglu, Ozdaglar, Tahbaz-Salehi, 2015; Acemoglu, Carvalho, Ozdaglar and Tahbaz-Salehi, 2012; Freixas, Parigi, and Rochet, 2000). Other applications can be found in: Bramoullé, Kranton, and D'Amours (2014) that examine social links that shape economic interactions, as in markets, and equilibria depend on network measures; Strogatz (2001) that analyzes complex networks in of nonlinear dynamics (2001); Helbing (2013) that studies connected, global networks of highly interdependent systems that are vulnerable to failure at all scales, with serious risks to society, the complexity and interaction in the network may become unstable; and König, Tessone, and Zenoue (2014) that develop a dynamic network model to explain the observed nestedness in real-world networks, their approach use stochastic stability to identify the networks to which the network formation process converges and find that they are nested split graphs. Finally, a fine introduction to modeling with network theory can be found in Ahuja (2017) and Oh and Monge (2016).

The IOTs quantify the flows of goods and services that are exchanged among industries and the most important producers and consumers in an economy. Because various tables are crafted in distinct levels of aggregation, the nodes usually represent a respective scale of economic activity. In standardized United Nations methodologies (UNSTATS, 2007), the information to construct an IOT comes from Supply and Use Tables (SUT), which are themselves collected from surveys that monitor what specific firms produced (and to whom their production was sold in the economy) as well as what they consumed (and which economic actors supplied them). These SUT are indeed at the level of the private firm, so their flows can be described as exchanges of goods and services between specific industries. When they are compiled into a symmetric IOT; however, such precision is lost into categories of providers and consumers grouped around common goods and services, themselves differentiated between primary and secondary products.

The list of common goods and services is standardized for easy comparison. In this way, the relationships established in a symmetric IOT are not the real ones between firms, but a reordering of these based on similar products. In other words, IOTs are just one model of an economy ordered following a certain criteria, not a literal map of the economy itself. This assumption is important to be considered when the IOTs are used to model trade between countries, and a relevant research question is to quantify how IOT models of trade differ from more individualized country-country databases of trade. 
486 REMEF (The Mexican Journal of Economics and Finance)

What makes Input-Output Tables of Trade of Raw Material Goods Peculiar Networks? The World and Mexican Cases

Therefore, in a highly aggregated IOT, the consumers and producers are less defined than the flows themselves. When ordering groups of goods and services together, some information is lost on who specifically produces and consumes. IOTs are, in this way, models of flows of goods and services more than models on producers and consumers. In a network analysis of IOT, this matters because various definitions of what a node is may lead to distinct outcomes. The node is usually defined by the level of aggregation given the United Nations standardized methodology. For example, a node could be the flow of economic goods and services between aggregates to the four digit level of classes, whereas in other classifications the node could be the end or beginning of a flow of economic goods and services at a more disaggregated level, much closer to the firm. Furthermore, this is one of the explanations of the particular network structure of an IOT when comparing with many other real life networks. Real life networks can share common properties of complex networks, while network structures of IOTs are highly aggregated categories of nodes that have in common primary flows of goods and services. The nodes may be more or less densely connected with each other depending on the level of aggregation.

The most important characteristic of an IOT is the flow or the edge of the network. What flows in an IOT are goods and services, usually in standardized monetary units. These do not flow in disordered or stochastic ways, but have a specific orientation. A farm will give its products to many sectors of the economy as food, for example, but a specific farmer will only sell its primary products (i.e., fresh meat) to a specific buyer or node in the network (i.e., household or butcher). When aggregating many such farmers and associating suppliers give form to the agriculture aggregate, which in this example becomes a source node, and other actors are aggregated as, for example, the food products sector, here the target node.

In the above example, an arrow represents the total output that a country's agricultural aggregate has provided for the food products aggregate. The arrow is usually in monetary units, which includes a combination of several quantities and the respective prices of the goods and services provided. In this manner, the resulting graph is bidirectional (it matters who provides and who consumes) and contains a high level of self-loops (in the example, a farmer providing a meat producer could be a self loop of agricultural aggregate to itself). The higher the level of aggregation, the denser the self-loops in some industries, since the number of industries that provide themselves with resources is more aggregated. In this way, the most important characterizations for IOTs are not necessarily the typical centrality measures (as will be presented below), but measures that mainly characterize the relative importance of traffic flow, for which the basic tool stock of network analysis is more scarce.

\subsection{The study of global trade through network analysis of IOTs}

The study of IOTs using network analysis is a growing subject, as there are already hundreds of investigations. However, those relevant papers to study global trade in general, and specifically raw materials trade, are much less numerous. Following is a summary of these, organized around the topic of interest and methodology utilized (i.e., network centralities applied, some of which are reviewed in the methodology section of this paper). In terms of analyzing key sectors, some works are: Alatriste (2014) studying several European economies with hubs and authority measures to evaluate impacts of shocks in trade as a function of many variables, including the relative importance of various sectors; Blochl et al. (2011) analyzing OECD economies in terms of random walk centrality and counting betweenness; and Muniz et al. (2008) measuring key sectors by the total effects exerted on the economy, the immediacy (a more or less direct tie by which the sector 
connects with the others), and its importance as a factor in transmitting effects throughout the network.

The investigations in terms of determining the general structure of an economy are: Carvalho (2013) examining the distinct structures that have been found by various IOTs analyses, and McNerney et al. (2013) and McNerney (2009) carrying out general analyses of world IOT, characterizing the statistical structure of the entire global network as one that can be approximated by a Weibull distribution, as well as the structure shared by OECD countries, respectively. Another important problem discussed in the literature is inequality in international trade, where a global trade network is dichotomized in two main groups, the core and the periphery. In this regard, Prell (2016), Prell et al. (2015) and Muniz (2011) complement a vast literature under the title of dependency theory and ecologically unequal exchange. All these authors are interested in analyzing the distribution of pollution and wealth among countries, studying how structural patterns of international trade give rise to emissions inequalities across countries, as well as its impact on mortality rates, and determining the relative position of each industry via global core-periphery models.

Beyond the two main groups stated above, there is a more diverse group of network analysis on IOTs, such as intra-regional trade, urban-rural dynamics or flows of pollutants, but these are not as directly relevant to the focus of this paper - a study of the trade of raw materials. Some exceptions include the IOT network analysis of the construction industry trade for Turkey (Kaya, 2017) and of the aluminum flows in the US (Nuss et al., 2016). This literature is associated to alternative methodologies such as Material Flow Analysis (Suh, 2005, 2009) where an important topic of future research is to explore the possibility of combining material flow analysis with network analysis and input-output tables, as suggested by Pauliuk (2015). However, to the knowledge of the authors, there has not been a published work either reviewing the application of the concept of network analysis on IOTs or quantifying with this methodology the trade of raw materials.

As was argued above, a central issue for network analysis of IOTs is a relative characterization of flows. In this sense, the literature is scarcer, and it is a relevant research question to see how much the aforementioned literature takes into account explicitly the nature of the flows or traffic. While in theoretical discussions or mathematical graph theory models the real life characteristics of the flows might be irrelevant, for some IOTs network analysis applied to real world problems and policy prescriptions, it is paramount to determine the nature and characteristics of the traffic in each network, as is suggested in the next paragraph.

Borgatti (2005) highlights the importance of making explicit the type of traffic involved for all network analyses in general. He characterizes flow in networks based on two dimensions: the trajectory taken by distinct types of traffic throughout the entire network and the transmission mode of traffic between each pair of nodes. For example, the author compares among several networks in terms of characteristics of flows. In networks measuring physical monetary flows (for example, an economy or a financial system network), the trajectory taken follows certain properties. The physical monetary unit (a coin or a bill) is indivisible and can only be in one place at a time. However, it is not proscribed from passing over the same link more than once. More precisely, it could easily move from A to B, B back to A, A to B again, then B to C and so on. From a graph-theoretic point of view, the bill traverses the network via walks rather than trails. As a result, the movement of money can be modeled as a Markov process. In contrast, in networks measuring used-goods (such as the input-output models), the trail is more relevant, or a sequence of incident links in which no link is repeated. Trails are distinguished from 
488 REMEF (The Mexican Journal of Economics and Finance)

What makes Input-Output Tables of Trade of Raw Material Goods Peculiar Networks? The World and Mexican Cases

paths -sequences in which not only links but also nodes cannot be repeated- and walks, which are unrestricted sequences. In this way, Borgatti argues, that the assumptions of some centrality measures would apply more to the network of physical money but not so much to the network of used goods, and vice versa. Such distinction gives way to a typology of flows and mode of transmission, for which the author argues that a given node centrality measure will be relatively more (or less) successful at measuring a specific network property if it correctly acknowledges (or not) the type of traffic flow for the problem analyzed. For example, Borgatti shows how Freeman's betweenness centrality (see below) works best when the network under consideration has traffic that will follow shortest paths and is transferred directly from a node to another node (i.e., a package that is delivered). It will not give the same optimal results for a network where the traffic drifts along longer or redundant walks and when it is transferred from one node simultaneously to many others, through parallel duplication (i.e., attitude influencing).

Additionally, the study of flows, as it is relevant to an IOT network analysis of trade of raw materials is an example of a problem where other methods of flow analysis, even from other disciplines like physics might prove useful. For example, Donner et al. (2017) revise several applications of complex network analysis on various flow systems such as trade, the climate system or the human brain. Some methodologies suggested by the author, like correlation networks or time series networks can also prove useful to measure flow systems, and it remains an open question how suitable they could be to quantify flows of specific goods and services. In the following sections, there will be a discussion of some flow properties as three distinct databases are compared, in order to highlight how IOT networks may differ from other networks.

\section{Methodology and Data}

The previous section showed some reflections on what are the best measures for a network analysis of IOTs, emphasizing on measures that capture peculiarities of flows that are idiosyncratic to IOTs. This section presents a methodology, which subsequently will be applied in following section. As mentioned before, there are two common objectives of network analysis: to determine a specific topology and to determine the relative importance of nodes, given this topology. Common examples of topologies in complex network analysis are scale free, small world, hierarchical topologies or distributions (Strogatz, 2001). The topology of a network is important because it is the macro property that determines which nodes are important, relative to others. Therefore, regardless of the network being directed or not, it is important to determine first the general topology of the network. Once this is done, the next step taken by many researchers is to analyze the nodes of the network in terms of the various centrality indices. Conventional centrality indices of node importance are: degree (Proctor and Loomis, 1951; Shaw, 1954), betweenness (Freeman, 1978), closeness (Hakimi, 1964; Sabidussi, 1966; Freeman, 1978), eigenvector (Pinski and Narin, 1976; Bonacich, 1987) and page rank centrality (Page, Brin, Motwani, and Winograd, 1999). Many other topological and sector analyses can be, of course, carried out, as vast as it is graph theory and other network methods. However, this paper will only focus on the above cited topology and node analysis.

In the following section, an exercise for three datasets will be carried out: one that is known beforehand will result in a scale free, hierarchical network topology, and IOTs. Two different IOTs are compared, one at a national level, and other at an inter-country level, so that similarities between them can also be ascertained. The purpose of this basic comparison is to highlight the particularities of the IOTs when compared with a network that follows commonly studied topological characteristics, as described above (Strogatz, 
2001). This comparison will provide some reflections on the appropriate application of such methods to IOT network analysis.

The IOT data utilized are from the World Input Output Database ${ }^{3}$ (Timmer et al., 2015) for the regional matrices (WIOT11) and the OECD 2011 database for the national one, for Mexico (OECD11mx). There are several databases in the web that follow the same topological patterns described by Strogatz, but the key characteristic criteria is to find one that is a digraph (its edges have a specific direction) and is as close as possible in terms of number of nodes (and thus, possible edges) as the world IOT. The resulting database (EMAIL) is a survey of e-mail exchanges in a real-life organization made up of 1,700 employees (Guimera et al., 2003). The choice of this EMAIL database is trivial, it does not matter which database is used, as long as it shows the topological characteristics defined above. The software utilized for network analysis is Cytoscape (Smoot et al., 2010).

\section{Comparison of three digraphs: what makes IOTs different?}

This section presents the results of the comparative analysis. A basic network analysis with the most common measures and centralities is carried out for all three datasets, and the results are evaluated, with the idea of highlighting peculiarities of IOTs and the relevance of their analysis with various centralities.

\subsection{General statistics}

As it can be seen in Table 1, one of the main differences between the EMAIL network and any of the two IOTs is the compactness of connection between the nodes, seen through various measures such as the number of edges and the degree or the density. The first network is less dense (0.009), which means that from all the possible connections between all nodes only 0.009 percent are actually connected. The second network regarding Mexico is completely connected. The total possible connections are 1056, and the network has 1078 links, with 22 nodes having self-loops. The third inter country IOT is less connected than that of Mexico but 55 times more connected than the EMAIL one with a density value of 0.495 . A related concept, the network clustering coefficient shows a similar trend, very low for the EMAIL database, and very high for the World IOT, even close to one for the Mexican network. IOTs tend to be highly connected and very dense, regardless of it being a national or a regional one.

Table 1. Network analysis with basic statistics of three digraphs

\begin{tabular}{|l|c|c|c|}
\hline Metric & Email & OECD11 mx & WIOT11 \\
\hline Number of nodes & 1,133 & 33 & 1358 \\
\hline Number of edges & 10,902 & 1,078 & 913,987 \\
\hline Average Degree & 19 & 65 & 1346 \\
\hline Average In Degree & 10 & 33 & 673 \\
\hline Average Out Degree & 10 & 33 & 673 \\
\hline Diameter & 8 & 1 & 3 \\
\hline Radius & 5 & 1 & 2 \\
\hline
\end{tabular}

\footnotetext{
${ }^{3}$ This paper uses the World Input Output Database under the hypothesis that the same methodological issues apply to other Multi Regional IOT. Countries considered in the WIOT (CODE) are: Australia, Austria, Belgium, Brazil, Bulgaria, Canada, China, Cyprus, Czech Republic, Denmark, Estonia, Finland, France, Germany, Greece, Hungary, India, Indonesia, Ireland, Italy, Japan, South Korea, Latvia, Lithuania, Luxembourg, Malta, Mexico, Netherlands, Poland, Portugal, Romania, Russia, Slovak Republic, Slovenia, Spain, Sweden, Taiwan, Turkey, UK, USA.
} 
490 REMEF (The Mexican Journal of Economics and Finance)

What makes Input-Output Tables of Trade of Raw Material Goods Peculiar Networks? The World and Mexican Cases

\begin{tabular}{|l|c|c|c|}
\hline Metric & Email & OECD11mx & WIOT11 \\
\hline Density & 0.009 & 1 & 0.495 \\
\hline Average shortest path length & 3.606 & 1 & 1.260 \\
\hline Clustering coefficient & 0.220 & 0.990 & 0.731 \\
\hline \multicolumn{2}{|c|}{ Source: Own elaboration with data from OECD and WIOT. }
\end{tabular}

Regarding other measures, Wasserman and Faust (1994) focuses on social ties between people such that the nodes are people, called an actor, as the central point of reference. Measures of interest in analyzing complex social networks are based in the concept of the "shortest path(s)", with the idea that in terms of efficiency, it would be best when anything that flows through a network would do so along the shortest path(s) available. In this sense, an interpretation of a path could be, according to Wasserman and Faust (1994), that the nodes are employees in an organization. If information originating with one employee could eventually reach all other employees, how fast and how many nodes it must traverse in order to get there (as well as possible barriers or bottlenecks for this information flow).

Clearly, when an indivudual obtains an advantage when receiving information first, a shortest path is highly preferable. One might also consider whether there are multiple routes that a message might take to go from one employee to another, and whether some of these paths are less "efficient" (Wasserman and Faust, 1994; p. 105). The key assumption in this measure is variability in terms of length of paths. If there are many paths and these are of different lengths, then it makes sense to measure the shortest ones that would give the most efficient form of information transmission. In this sense, the average shortest path length of 3.606 in the EMAIL database means that any employee in this firm has sent or received an EMAIL passing through approximately three-to-four other employees, all connected to form a shortest path of average length 3.6, but in the context of several other diverse possibilities of length if interconnected. By contrast, for both IOTs, the shortest paths tend to be close to one (Mexico is 1, and in the WIOT is 1.2). This means in the context of an IOT that every sector of the model of economy that is represented by an IOT delivers its goods and services (or receives goods and services from) to other sectors mostly through paths of length one, without intermediaries, and there is little variability in this form of connection between any nodes. In other words, the IOTs are usually structured as a one-step network. In this way, when a centrality from network analysis is based on the assumption that traffic flows along a range of length of paths, the IOTs will provide peculiar results when all their shortest paths have length one.

Related to shortest paths is the diameter of the network, which measures the longest shortest path between the nodes and in this way gives an idea of how "wide" a network is. It is not surprising to find that for the EMAIL database, there is a quite a long average shortest path (of length 8) when compared to the IOTs (length 1 for the Mexican case and 3 for the World IOT). What this means is that every node of the IOT is connected by shortest paths of a lower order of magnitude, contributing with its characterization of very densely connected networks. The relevance of this measure may be distinct according to the problem at hand. An important question in the case of an IOT is: What does the shortest path length imply in terms of economic significance? In a general view, any path in an IOT exemplifies a proxy for a chain of production, although as it has been presented above, it is a rather artificial one. Primary production sectors offer their part of their goods or services to another type of sectors, some of these with higher value added, which in turn offer part of their goods or services to another type of sectors. If this is the 
significance of the chains of steps into paths of the IOT network, then an issue can be made against looking for shortest paths. On the contrary, a longest path that is not an iteration within the network would arguably entail higher value added in integrated chains of production. The IOT is not necessarily a map of value added; however, this idealized scenario is an exercise of extreme arguments that points toward questioning the basic assumption of shortest paths built into some network analysis tools when utilizing IOT. To see what different types of paths there are in the two IOTs studied, the next subsection looks at their distribution.

\subsection{Centrality Distributions}

While the above measures are related to nodal properties, it is usually more telling in network analysis the distributions and variability in the data. Figure 1 shows the distributions of the lengths of shortest paths of the three databases.

Figure. 1 Distributions of shortest paths lengths

EMAIL

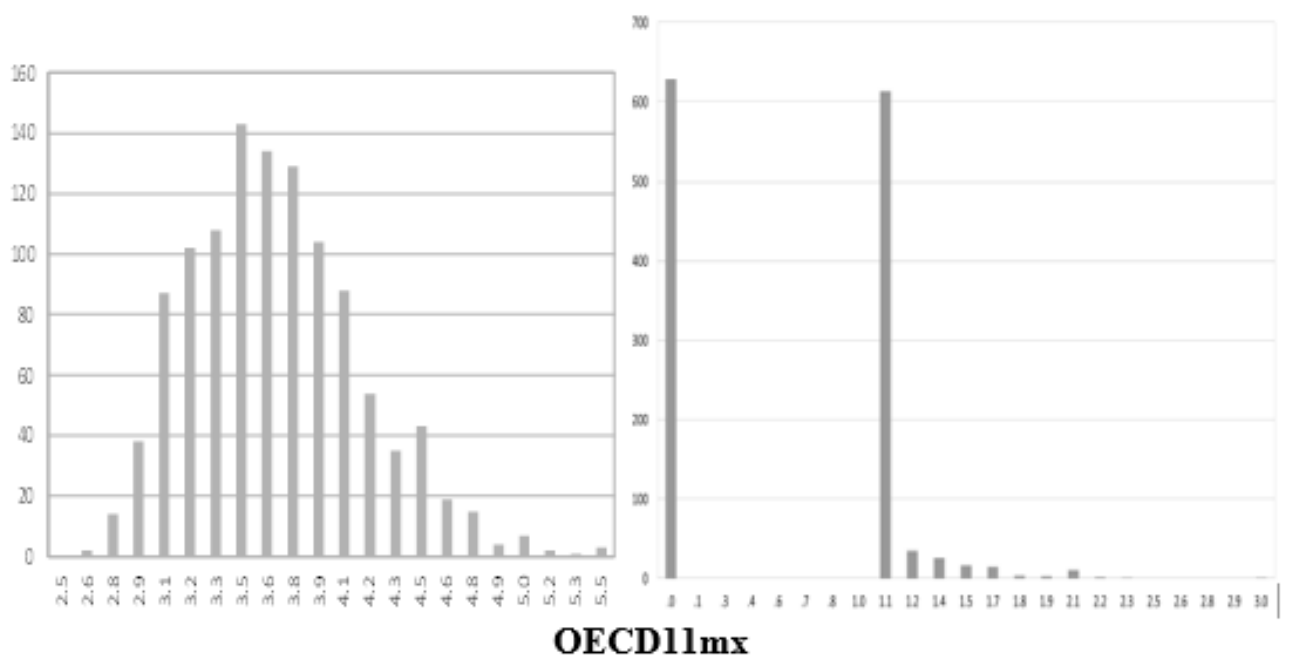

OECDIlmx

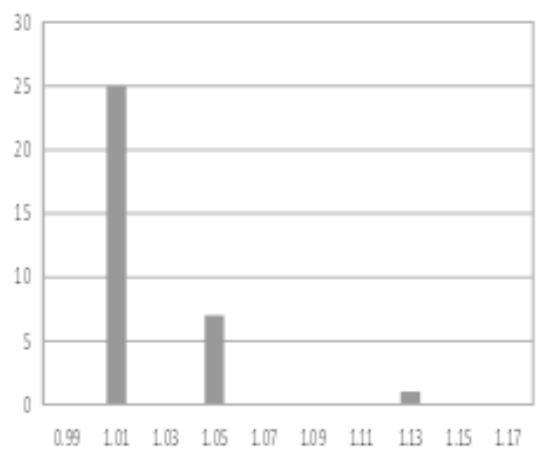

Source: Own elaboration with data from OECD and WIOT. 
492 REMEF (The Mexican Journal of Economics and Finance)

What makes Input-Output Tables of Trade of Raw Material Goods Peculiar Networks? The World and Mexican Cases

As seen in the three histograms above, indeed longer shortest paths are rather rare in all three cases, but mostly for the IOTs. Firstly, the range of length for the shortest paths is different in the two cases. For the EMAIL database, the shortest path length range goes from a minimum of 2.6 to a maximum of 5.48 , whereas for the IOTs such range starts at 0 and ends at 2.9 for the world IOT and respectively 1 to 1.125 for the case of Mexico. A limited range of possible lengths is also accompanied with very little variability, by far the most important characteristic of the IOTs in network analysis. Compared to the normal distribution of shortest path lengths of the EMAIL database, the middle WIOT has two most frequent average shortest path lengths, 0 and 1.1, respectively, with frequencies of 629 and 614. For the Mexican IOT, the most frequent average shortest path length is around the value of 1.03 , with 25 nodes (out of 33) having this measure.

Another common distribution of network analysis is the degree distribution, which points to the total number of neighboring nodes that any given node is connected to, and which is also studied as in-degree and out-degree for incoming and outgoing links (mails or goods and services), respectively, with other nodes. One first characteristic to highlight is the relationship between the total number of nodes and the average degree of the rest of the nodes. As Table 1 showed, the EMAIL digraph has 1,133 nodes, whereas the average degree is 13 , and 10 for the in/out degree. This is contrasting with the data from IOTs where the total number of nodes for Mexico and the world are respectively 33 and 1358, and the average degrees are 65 and 1346, whereas the in/out degrees are 33 and 673, respectively. That is, both IOTs tend to be densely connected, or have a higher degree in proportion to the total number of nodes where all nodes are connected to almost every other node of the entire structure. This is evident in the respective degree distributions, as shown in Figure 2.

The first row of graphs in Figure 2 shows the differences in degree if it is assumed that the network is undirected. The right-side distribution for EMAIL data shows a typical scale free structure of complex networks, where a few nodes or hubs (in this case, only one, the farthest right) have a really high degree (142 neighbors) and most nodes have lower degrees (the highest frequency bar at the extreme left), mostly 2 neighbors. The distributions for the two IOTs, however, are far from having a scale free structure, and are themselves not similar. In fact, their distribution tends to be skewed to the right, the opposite direction of what is expected from a scale-free distribution. For the Mexican graph, most nodes have a high degree between 55 and 65 , while the middle WIOT has two distinct peaks of degree values, around 700 and at the highest of 2000. Since degree is the sum of in-degree plus out-degree, the following paragraph will show how these IOT results are composed. Differentiating this measure into in and out degrees, the second and third rows in Figure 2, respectively, present a more similar picture for the IOT, which is different than that from the EMAIL database. The EMAIL database distributions of in and out degree present again a scale-free distribution. Most nodes have a lower in and out degree. As the number of nodes decreases, the in/out degree increases. Thus, very few nodes have a really high in and out degree. Only one node has the highest possible in-degree of 71 (or only 1 person receives e-mails from other 71 persons in the network), and only one has an out degree of 71 , in the same manner. 
Figure 2. Distributions of degree

Top row: degree; center row: in-degree; bottom row: out-degree Left: EMAIL; center: WIOT11; right: OECD11mx
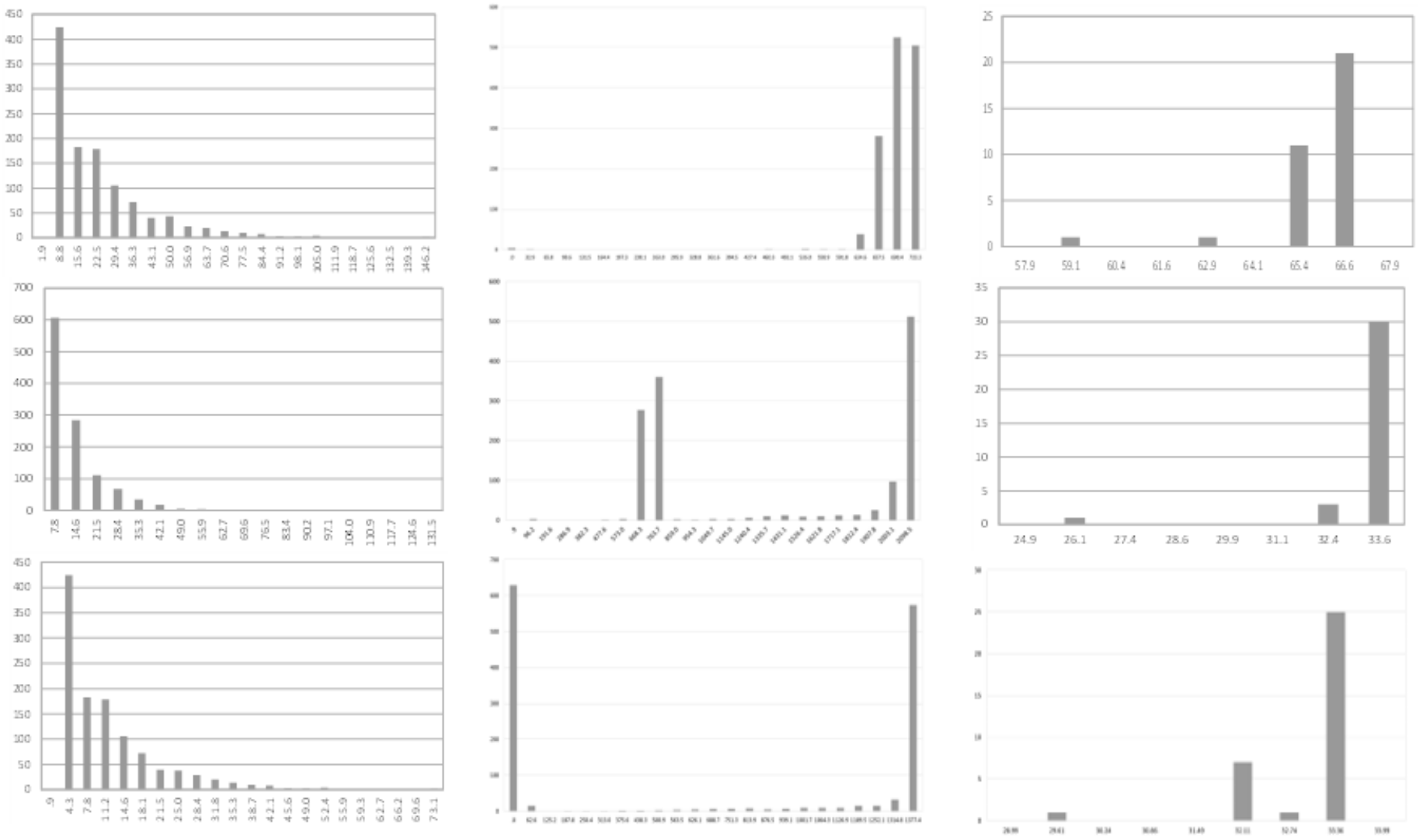

Source: Own elaboration with data from OECD and WIOT. 
494 REMEF (The Mexican Journal of Economics and Finance)

What makes Input-Output Tables of Trade of Raw Material Goods Peculiar Networks? The World and Mexican Cases

For both IOTs, the structure of these distributions are again opposite. A lot of nodes have a high in/out degree, and very few nodes have a low value of these measures. Even when the middle in-degree WIOT11 distribution seems to encompass some variability around a mean, the range comparisons between the in-degree of the EMAIL database and the WIOT is more telling. For the EMAIL database, most of the in-degree measures are found between a range of 1 and 56 inbound neighbors, whereas for the WIOT this range is from 624 to 723 in-bound neighbors. For the Mexican IOT, the variability is even more constrained, when most nodes have an in-degree of 32-33 neighbors.

Of particular interest are the differences of distributions between the in-degree and out-degree distributions, for any network, but most importantly for the IOT analysis. The reason being that it makes a difference when a sector supplies many other sectors in the economy, as it could be the case on a primary product sector, and when raw materials as components of the goods in flow, as opposed to when a sector is supplied by many other sectors in the economy. This difference is of more importance in an inter-country IOT where countries that tend to supply raw materials to the world would arguably have the highest out-degree of the network for the relevant sectors and where as the highest consumers of goods and services in the world would have the highest in-degree. The second and third row in Figure 2 shows these differences. For the EMAIL database, both in and out degree show a scale-free topology, which suggests a rather hierarchical structure in the organization under study, where very few people have direct email contact with the rest of the employees. For the WIOT, however, one main difference stands out, even when both in and out degree distributions are mostly skewed to the right, meaning that most sectors have a high number of direct suppliers and supplied, the frequency of zero neighbors is higher for the out-degree (629) than for the in-degree (4). What this means is that most sectors in each country are supplied by many other sectors, both in and outside of the same country, whereas half of the country-sectors do not supply any other country sector in the world. This is an important differentiation in itself, offering some variability to an otherwise rather homogeneous distribution, but also in economic terms, because it allows to see which sector-countries supply the most to the rest of the global commerce network, as opposed to those that do not supply any sector/country at all (Table 2). Such variability is lost in the Mexican IOT, where all nodes are as much suppliers as are supplied in the network, probably a result of the high aggregation of flows.

Also, Table 2 shows the twenty sector-countries with the highest out-degree. As is evident, most of these nodes offer either services or raw materials to other sector-countries, so that a first hypothesis is that out-degree can serve to differentiate sectors in the primary production and service category from those of other categories. However, important exceptions are also sector-countries that have a high out-degree and belong to the secondary industrial sector, such as the transport equipment industry from India (IND), since having a high out-degree could point to this sector being an important provider in global chains of production. The objective here is only to explain this variability in out-degree, and an interesting topic for further research is to go deeply into the specifics of such differences.

These aforementioned basic structural characteristics build into more complex ones, two of which aim to further capture variability among nodes and for which the shortest path is the fundamental building block: a) betweenness centrality quantifies of all the shortest paths between any nodes, the ones that pass through a specific node; b) closeness centrality quantifies which nodes are closest to most other nodes, as captured by the shortest paths between any node and all its neighbors; and c) clustering coefficient centrality describes how the different nodes tend to cluster or group to other nodes. In 
this way, it is not surprising to find marked differences in the distributions of each of these centralities for each of the three datasets analyzed, as shown in Figure 3.

Table 2. Highest Out-degree nodes in WIOT $11^{4}$

\begin{tabular}{|l|c|c|c|c|}
\hline \multicolumn{1}{|c|}{ Country code/Sector } & Degree & In-degree & Out-degree & Category \\
\hline FRA Public Admin and Defence; Compulsory Social Security & 2042 & 688 & 1354 & Administrative \\
\hline CZE Other Non-Metallic Mineral & 2055 & 701 & 1354 & Raw materials \\
\hline GBR Other Community, Social and Personal Services & 2058 & 704 & 1354 & Services \\
\hline GBR Renting of M\&Eq and Other Business Activities & 2058 & 704 & 1354 & Raw Materials \\
\hline HUN Other Non-Metallic Mineral & 2058 & 704 & 1354 & Services \\
\hline $\begin{array}{l}\text { BRA Sale, Maintenance and Repair of Motor Vehicles and } \\
\text { Motorcycles; Retail Sale of Fuel }\end{array}$ & 1966 & 613 & \multirow{2}{*}{1353} & Services \\
\hline $\begin{array}{l}\text { BRA Retail Trade, Except of Motor Vehicles and Motorcycles; } \\
\text { Repair of Household Goods }\end{array}$ & 1967 & 614 & \multirow{2}{*}{1353} & Services \\
\hline $\begin{array}{l}\text { BRA Wholesale Trade and Commission Trade, Except } \\
\text { of Motor Vehicles and Motorcycles }\end{array}$ & 1967 & 614 & \multirow{2}{*}{1353} & Administrative \\
\hline BRA Public Admin and Defence; Compulsory Social Security & 1986 & 633 & 1353 & Raw materials \\
\hline CAN Leather, Leather and Footwear & 1995 & 642 & 1353 & Services \\
\hline CAN Education & 1998 & 645 & 1353 & Raw materials \\
\hline CAN Coke, Refined Petroleum and Nuclear Fuel & 2000 & 647 & 1353 & Raw materials \\
\hline CAN Other Non-Metallic Mineral & 2002 & 649 & 1353 & Raw materials \\
\hline IND Basic Metals and Fabricated Metal & 2002 & 649 & 1353 & Raw materials \\
\hline IND Pulp, Paper, Paper, Printing and Publishing & 2003 & 650 & 1353 & Raw materials/Manufactures \\
\hline IND Rubber and Plastics & 2003 & 650 & 1353 & Manufactures \\
\hline IND Transport Equipment & 2003 & 650 & 1353 & Manufactures \\
\hline GRC Electrical and Optical Equipment & 2004 & 651 & 1353 & Manufactures \\
\hline GRC Machinery, Nec & 2004 & 651 & 1353 & Services \\
\hline GRC Transport Equipment & 2004 & 651 & 1353 & \\
\hline
\end{tabular}

Source: Own elaboration with data from OECD and WIOT.

The first row in Figure 3 shows the distribution of betweenness centrality values for all nodes for the three datasets. In the far left, the EMAIL database shows a scale-free distribution of betweenness centrality, similar to the degree distributions shown in figure 2. What this means is that, as was discussed above, there are few key nodes that are important because they are hubs of the network, or nodes that connect to most or all of the other nodes. An additional property of some complex networks; however, these same hub nodes serve as bridges for information flow for the nodes rest in the network, or have high betweenness centrality values. Thus, the correlation between degree and betweenness centrality in complex networks can be high, in this case 0.865 .

The middle upper distribution is the betweenness centrality measures for the world IOT. At first glance, the distribution looks scale-free, as in the EMAIL database; however, a closer look describes again very little variability with most nodes having very low values of betweenness centrality. In contrast, the correlation between degree and betweenness is lower, of only 0.472. The reason for this is that, as in Figure 2, most nodes had a very high degree (so there are not key nodes in terms of their connectivity to others), and these nodes have also low values of betweenness centrality. For the Mexican IOT, even this pattern is lost, with no clear division between nodes, except for the high frequency of very low values of betweenness centrality. The peculiarities of the results in betweenness centrality distributions for IOT network analysis are without doubt a result of the lack of variability in shortest paths. Because betweenness centrality seeks to measure how many of the shortest paths pass through a specific node when most paths are themselves shortest paths of length one, then very few nodes (if any) will have a high value of this measure, meaning that all small paths pass through all or most nodes.

\footnotetext{
${ }^{4}$ For the abbreviations of countries utilized in this study, see the Appendix.
} 
Figure 3. Distributions for various centralities:

Top row: betweenness; center row: closeness; bottom row: clustering

Left: EMAIL; center: WIOT11; right: OECD11mx

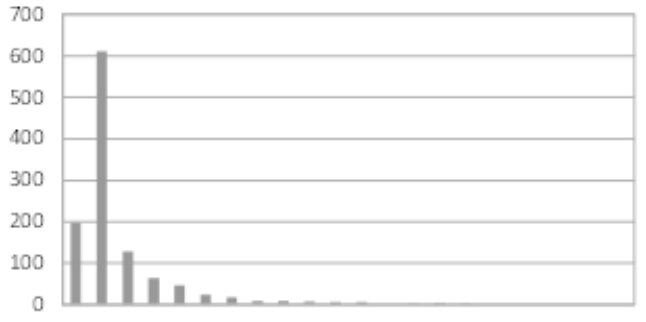

000.004 .008 .012 .016 .019 .023 .027 .031 .035 .039
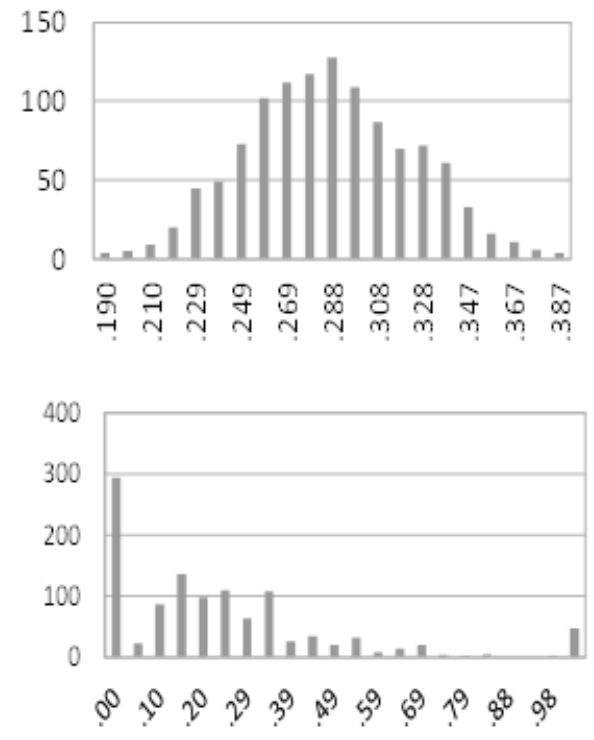
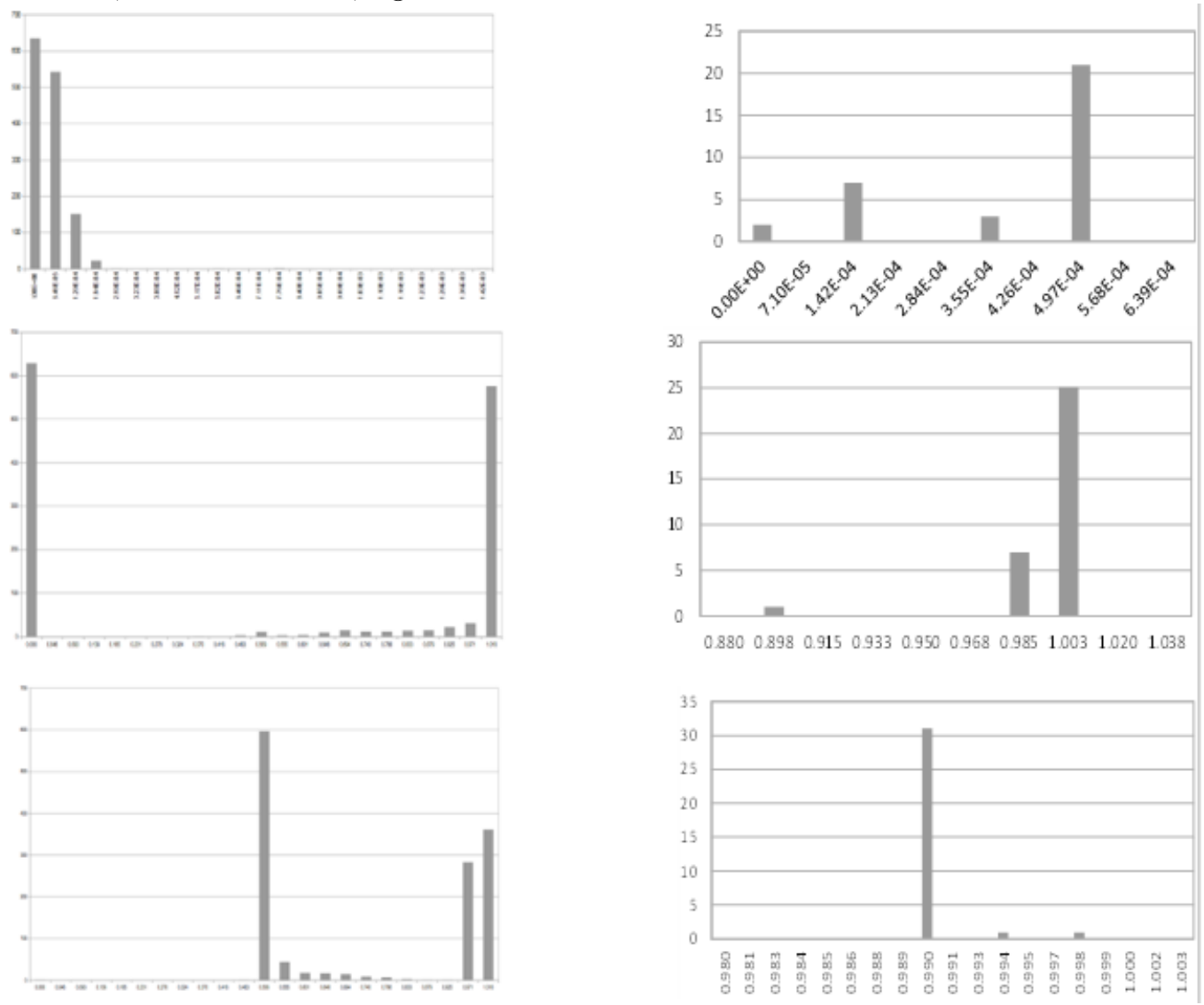

Source: Own elaboration with data from OECD and WIOT.

. 
For closeness centrality, in the second row of Figure 3, the results are similar. The EMAIL database shows a relatively normal distribution for closeness centrality, with an average of 0.281 , which points to most nodes being relatively close to most other nodes. In contrast, the WIOT shows the two highest frequencies, on the left is a bar representing 629 nodes with a closeness centrality of zero, and in the right is a bar representing 576 nodes with a closeness centrality value close to one, or 0.999 . This signals a highly clustered network, where in the center one-half of the nodes relate to each other very intensely (probably through both in and out degree) and the rest of the network is rather sparse. The Mexican IOT is similar to the first group in the WIOT, in that most nodes (32 of 33) have closeness values very close to one.

The last row in Figure 3 shows the respective results for clustering centrality. The EMAIL database shows a distribution that is not as scale-free or normal as the previous ones, but still shows a lot of variability, with a range of clustering coefficients that spans several frequent values between 0 and 1 . The most frequent values, however, are closer to zero, which as in a scale-free distribution, denotes a network where most of the nodes are sparsely connected in small communities to other nodes, whereas there are few nodes (47 out of 1133) with the highest clustering coefficient of one. The combination of the previous measures with this one gives a possible hierarchical typology. Few nodes in the network are hubs (have many neighbors), from which most of them serve also as bridges to other peripheral, less central nodes, which tend to be surrounded by small communities of their own.

Not surprising at this moment, the story is not a clear-cut for both of the IOT. The lower middle distribution shows a WIOT with three highest frequencies: very close to one at the right side and one in the middle, for 597 nodes with a clustering coefficient close to 0.500 . This pattern tells a similar story of the one found for the closeness coefficient. There is a group of nodes (about half of the network) that are more highly connected among them, therefore being more central to the network, and thus forming a more tightly knit community than the rest of the nodes. This is likely the main result found in above cited works of center-periphery trade networks. For the Mexican IOT, the clustering centralities also tell the same story as the previous ones: most of them have values close to one, which point to a very tightly connected network, with little deviation in other topologies.

\subsection{Prestige Centralities}

As discussed in the previous subsection, in and out degree is a useful measure for network analysis of IOTs, because it can serve to differentiate categories of sectors such as primary production or service oriented, but important to the objective of this research, because it can give way to a quantification of relative importance in the participation of a country in global raw material trade. The next group of centralities is related to in and out degree, or based on prestige or influence. The most common used being the Eigenvector centrality and its relative Page Rank. These centralities, along with the related tool of Hubs and Authorities, are calculated based on the relative amount of in and out degree values for all the nodes. Although related, there a nuanced differences among the three centralities: Eigenvector centrality captures the relative importance of any node, in terms of how it connects to other nodes with high degrees; Page Rank captures the same properties as eigenvector centrality, but with a difference of scaling and the choice of the eigenvector; while Hubs and Authorities capture the property of nodes that refer other nodes, or those that are referred to by others, respectively. 
498 REMEF (The Mexican Journal of Economics and Finance)

What makes Input-Output Tables of Trade of Raw Material Goods Peculiar Networks? The World and Mexican Cases

As discussed above, the stated measures are particularly relevant to IOTs, since they take into consideration the relative amounts of in- and out-degree for each node, a probable proxy for what Hirschmann (1958) called backward and forward linkages, or supplier industries vs producing ones. In fact, the way to calculate in- and out-degree (the respective sum of columns and rows of the adjacency matrix) is similar to the way to calculate important coefficients in IO analysis (i.e., Rasmussen-Hirschman or Chenery-Watanabe coefficients). For weighted matrices, the in- and out-degree convert into the in and out strength; or the sum of rows and columns of the weighted adjacency matrix.

Figure 4 shows the distributions for Eigenvector and Page Rank centralities. Once again, the distributions are different between the EMAIL dataset, the Mexican IOT and the worldwide one. The distributions for both measures in the EMAIL database are very similar, and with a high amount of variability. In fact, they also resemble the distributions seen in the last section for in- and out-degree, which points to the similarity between prestige measures and in- and out-degree. The correlation between in degree/out degree and authorities/hubs is: 0.88 for the EMAIL database, and 0.99 for both of the IOTs. The same similarity is found between the in and out degree for the Mexican database and the respective prestige measures shown in Figure 4. However, one main difference between in and out degree and the prestige measures is found for the inter-country IOT. The eigenvector centrality for this database, in the first row, middle position, is quite different from the corresponding distribution of in degree. An increase in variability in the calculated values of eigenvector centrality for each node results in a smoother difference between each class. Nonetheless, the same problem as in-degree persists. That is, many nodes have high values of this centrality, making it hard to differentiate important nodes based on high values.

The changes from calculating the more tuned Page Rank are shown in the middle of the bottom row. A more normalized distribution results, with the important characteristic of separating a group of nodes with higher values of this centrality. ${ }^{5}$ The remaining aggregates of the entire world have values of Page rank in the logarithmic scale, ranging from 0.0004 to 0.0009 . Why such aggregates on these countries have the highest Page Rank values in the WIOT of 2011? Is indeed a good question for further research.

\footnotetext{
${ }^{5}$ The aggregate of Transportation of India (0.0012), Renting of MEq and Other Business Activities of India (0.0011) and Real Estate Activities of France (0.0011).
} 
Figure 4. Prestige centralities

Top row: Eigenvector; bottom row: PageRank

Left: EMAIL; center: WIOT11; right: OECD11mx
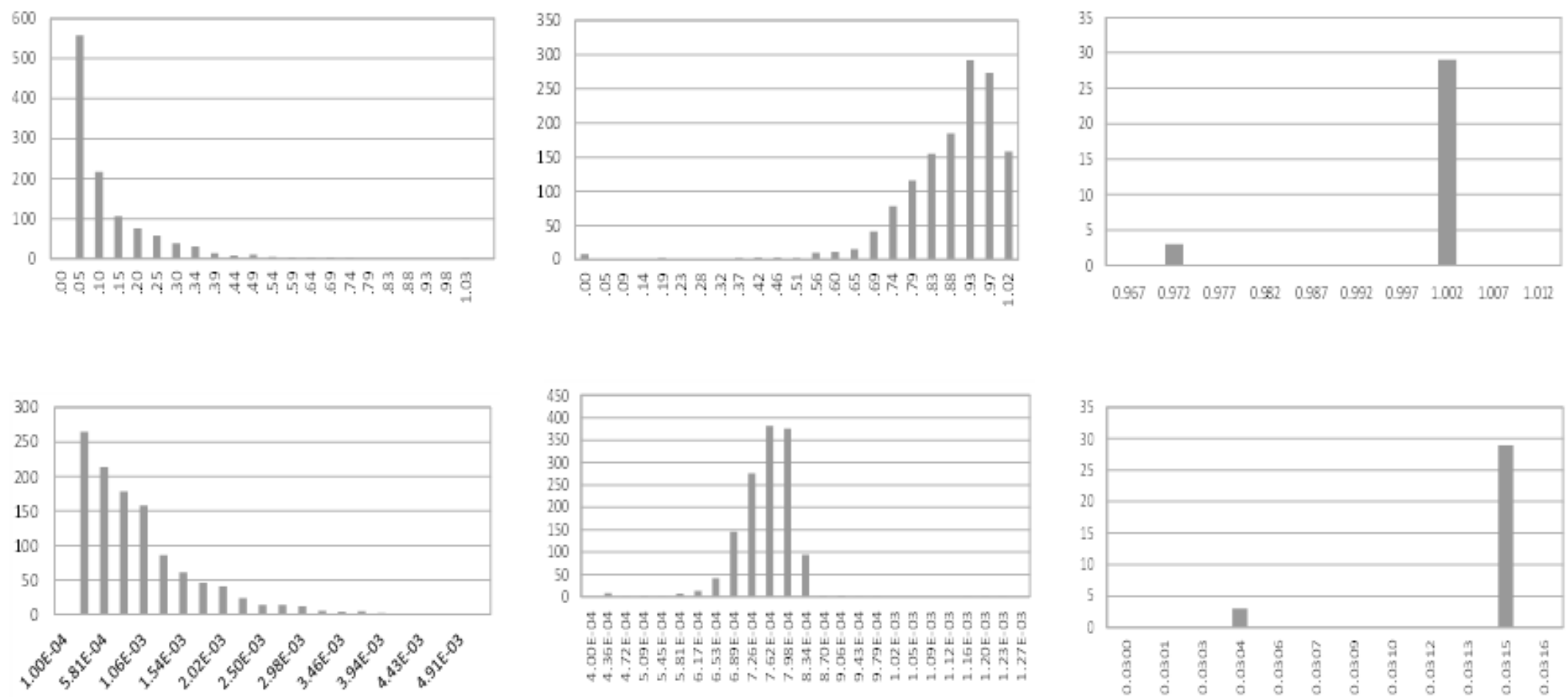

Source: Own elaboration with data from OECD and WIOT. 
500 REMEF (The Mexican Journal of Economics and Finance)

What makes Input-Output Tables of Trade of Raw Material Goods Peculiar Networks? The World and Mexican Cases

The distributions for Hubs and authorities are not shown, since they do not add new variability. In this case, the EMAIL and OECD ones are very similar to the respective in and out degree ones, and the WIOTs authorities distribution is very similar to the eigenvector one showed in Figure 4.

The first step in reviewing the main topological characteristics of the three datasets has been carried out with the important differentiation among the three datasets by considering different measures, and identifying certain key nodes for various measures. However, one main setback is that none of the previous calculations incorporate any information about the magnitudes of each flow, which is half of the value given by IOT. Having a portrait of the topology of the network without weights is necessary, but insufficient to a full network analysis of the IOT properties. The measures that incorporate weights are, to the best of our knowledge, still pending to be characterized and standardized. Several authors offer distinct approximations for each of the centralities studied above, and it is beyond the scope of this investigation to evaluate the pros and cons of each. Since weights do matter, following is a weighted degree analysis of the three sets, a common weighted measure in network analysis.

\subsection{Weighted Degree Analysis}

There have been already some applications of weighted network analysis for IOT in the literature, and it would be a further topic of research to review in detail these applications, specifying the pros and cons of each of the measure for the specific cases of national and multiregional IOT. Weighted degree can be calculated using different indices that contain in a standardized manner the degree of each node (or number of neighbors) plus the weight of each of the edges for these neighbors. In this way, a high weighted degree of a node could be due to any combination ranging from a higher amount of neighbors with low edge weights to the opposite extreme of lower amount of neighbors with high edge weights. Figure 5 shows the respective graphs for weighted degree distributions of the three datasets. The first columns are the same as the respective non-weighted Figure 2, since the EMAIL database is not a weighted network, or it has zero weights for each of its edges. 
Figure 5. Prestige centralities

Top row: Eigenvector; bottom row: PageRank

Left: EMAIL; center: WIOT11; right: OECD11mx
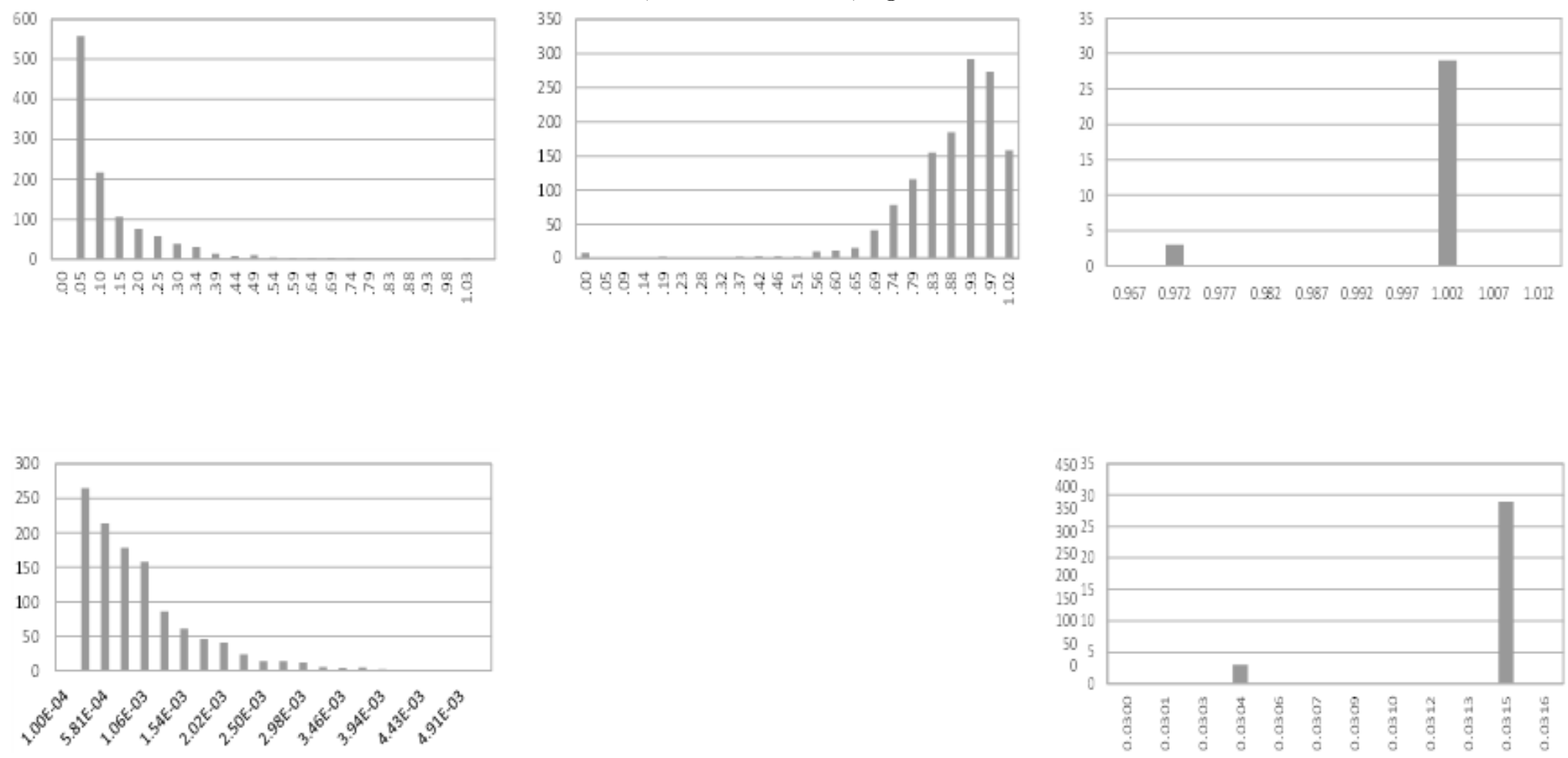

Source: Own elaboration with data from OECD and WIOT. 
502 REMEF (The Mexican Journal of Economics and Finance)

What makes Input-Output Tables of Trade of Raw Material Goods Peculiar Networks? The World and Mexican Cases

By contrast, the weighted distributions for the Mexican IOT have increased in variability, as have those of the WIOT, compared to their respective non-weighted ones. Something gained by calculating this index for both IOTs is the distinction between sectors or aggregates with higher values. The sectors with the highest in- and out- degree in both the Mexican IOT are shown in Table 3. Mexico's most important sector in terms of weighted in-degree is the food products, beverages and tobacco aggregate, meaning that this sector either has many other sectors as suppliers or the ones that it has have relatively higher monetary value than other sectors. A more detailed look at the two variables could give insights to what makes this sector central. On the other hand, the aggregate with the highest value of out-degree for Mexico was the Wholesale and Retail Trade, Repairs. In the case of the WIOT, there are two countries that make most of the top five positions in terms of in and out degree China and the United States. China's Electrical and Optical Equipment aggregate has the highest in-degree, (or most suppliers or supply intensities), as well as the highest out-degree (or most consumers or consumer intensities).

Table 3. Top five sectors or aggregates in terms of weighted in and out degree, for both the OECDmx11 and the WIOT 11 databases (see codes in Appendix)

\begin{tabular}{|l|c|l|c|l|l|l|c|}
\hline \multicolumn{4}{|c|}{ Weighted in-degree } & \multicolumn{3}{c|}{ Weighted out-degree } \\
\hline \multicolumn{1}{|c|}{ OECDmx11 } & \multicolumn{2}{c|}{ WIOT11 } & \multicolumn{2}{c|}{ OECDmx11 } & \multicolumn{2}{c|}{ WIOT11 } \\
\hline FOOD & 87,601 & ELECTRCHN & $1,463,891$ & WHOLESALE & 119,515 & ELECTRCHN & $2,171,840$ \\
\hline CONSTR & 76,448 & FOODCHN & 723,671 & RANDD & 81,209 & FINANCUSA & $1,691,116$ \\
\hline WHOLESALE & 62,072 & FINANCUSA & 712,068 & MIN & 63,337 & CHEMCHN & $1,131,411$ \\
\hline MOTOR & 56,601 & CHEMCHN & 640,582 & COKE & 51,228 & ELECTRRoW & 889,635 \\
\hline COMP & 51,978 & FOODRoW & 616,714 & CHEM & 49,396 & AGRCHN & 849,042 \\
\hline
\end{tabular}

Source: Own elaboration with data from OECD and WIOT.

The above paragraphs showed that weighted analysis adds some structure or variability to the IOTs, where other measures do not succeed in this manner. It is possible to distinguish more important or central nodes from the others by considering the weights. However, much remains to be done in the search for other methods that infer the structure of IOTs, seeing the weighted traffic in the network, especially as it changes in time. The next and last section will give a review of the main results obtained as well as directions for future research.

\section{Conclusions}

The main purpose of this research has been to make some methodological reflections on the particularities of an IOT when compared to a network with a scale free distribution and hierarchical organization. Having revised various tools of analysis of graph or network theory in the previous sections, one main conclusion is that IOTs are a very particular type of graph, for which the studied measures cannot result in satisfactory conclusions, or which have to be adapted in order to give a fragmented picture of the whole network.

Even when an IOT can be used to answer many questions, each IOT can require specific methods of graph theory; there can be important generalizations to guide future research on the subject. Clearly, every generalization that is made for one problem in mind will be different when the underlying problem changes; network analysis can have theoretical abstract methodologies, but when it is applied to real world problems, the characteristics of flows and nodes must be described and taken into account for its successful implementation.

This research also focused on studying trade in raw materials with a network analysis of IOTs. As is described in some references (Wiedmann et al, 2015 for a good summary) and under several lines of research (such as the natural resourse curse, or extractivism), 
there is a current debate over whether the income generated from the sales of raw materials in the global market and invested nationally promotes or damages economic growth. The debate is fostered out of complex causalities, but also from inadequate measures. Most measures tend to be nationally based (such as the percentage of raw material exports in total exports) and fixed to a specific time (usually, a year, or a proportion); they do not incorporate more relative comparisons with such measures. It is not straightforward to say, for example, that Mexico is more "extractivist" than Brazil, because they are national based and do not incorporate change in time in several years).

Network analysis of a multicountry IOT is a good candidate for a methodology to incorporate relative country differences of trade in raw materials, in terms of quantities and change in time. In this view, the question for future research is: what is the best set of tools from graph theory that can help differentiate from all the countries, those that export raw materials? From the ones revised, out-degree of the WIOT gave results that go in this direction, as would be expected, since as discussed above, sector-countries that have most values out-degree (hence are the utmost suppliers to the global trade) will likely tend to fall in the service or the primary production category.

Supplying the world economy with raw materials alone is not itself a sufficient criteria to fall into a category of extractivist countries. Other important considerations are the revenues obtained through exports, as well as the intensification of these revenues in a relatively short span of time. Gladly, the IOTs are calculated in total revenues (quantities supplied times a specific price), which are precisely what is considered as weights in this analysis. However, when this research presented the studies for weighted out-degree in the WIOT, there was an evident problem of scale, where the weights of China and the United States dominated the results. A finer analysis is pending, standardizing the production of each sector-country by total throughput of production, or GDP, as is done for national IOTs with the technical coefficients. Nevertheless, the problem of adequate categorization with weighted out-degree would remain in terms of the methodology used for calculating the centrality. In this way, a good result would be a weighted out-degree measure that does not leave much uncertainty about the relative pondering of degree and weights. Even better, weighted prestige centralities would give finer detail on which sector-countries are the suppliers to world trade. To the best of our knowledge, there has been no article on network analysis of trade of raw materials that uses this type of measures, a promising line of research.

Needless to say, there are many other centralities and topology characterizations not included in this research, which perhaps could give light to the problem of measuring raw materials trade. Similar to finding a needle in a hay stack, the problem is not so much a lack of resources, but a vast amount of methodologies that have been devised for specific academic niches, that are waiting to be translated into other ones, such as trade: motif analysis, dynamic network analysis, and Environ Analysis are but a few of these with much promise to give light to the current research question. Of help in this search would be delimiting what the centrality must do for optimal categorization. First, it must separate countries that are "in one end" of the network in terms of economic production: raw materials are the basis for many chains of production, at both national and global level, and the property that they are the beginning of many paths in the network must be able to be identified and separated properly.

Second, it must separate "normally centrally distributed" quantities of extraction (for example, the United States and China have vast amounts of raw material production, and are not necessarily considered extractivism in the relevant literature, as are Australia or Brazil), from outliers. To do this, the idea of distribution is a central tool, so that the 
504 REMEF (The Mexican Journal of Economics and Finance)

What makes Input-Output Tables of Trade of Raw Material Goods Peculiar Networks? The World and Mexican Cases

measure proposed could measure some part of the skewness of the raw material production distribution, or how big the tails are, and with this information, decide whether a country is more or less extractive than the others. In other words, each node has a distribution of in-degree and out-degree for every neighbor it has. A graph theory centrality would distinguish nodes based on some aspects of these distributions in such a way that when the distributions measure weights or intensities of raw material flows, the right tails of the distributions of each node would be more frequently visited. A good candidate for this analysis is random walk centrality, as studied for other objectives (Blöchl, Theis, Vega-Redondo, and Fisher, 2011) and a possible future research topic for the case of an IOT network analysis of raw material trade.

Thirdly, a time comparison is an intrinsic part of extractivism, both in quantities and in short periods of time, would make a country member in the category of intensified raw material production. Usually, network analysis concerns itself solely with static systems (topologies) that do not change in time; however, more commonly time comparisons are being carried out (in dynamic network analysis or correlation network analysis) so that these methodologies could promise to measure better an intense supply of raw materials to the global economic system in several time comparisons.

Network analysis, as it has been discussed, is full of methodologies that have proven to give very intuitive, sometimes qualitative, yet very useful descriptions of relational datasets and systems. The application of this method to an IOT has been a major leap to better understand economic systems, both at the national and the global level, which promises to get better as newer, more optimal network methodologies are devised, adapted to the peculiarities of IOTs. This paper has presented several analytical tools to give light to the idiosyncrasies of IOTs, and hopefully it will stimulate researchers to adopt, or even create, ad hoc methods to take most advantage of the information contained in an IOT. With the idea that having no model is worse than having an imperfect one, the disadvantages of network analysis of IOTs outlined in the previous paragraphs aim at this ultimate objective.

\section{References}

Acemoglu, D., Ozdaglar, A., and Tahbaz-Salehi, A. (2015). Systemic risk and stability in financial networks, American Economic Review, 105(2), 564-608.

Acemoglu, D., Carvalho, V. M., Ozdaglar, A., and Tahbaz-Salehi, A. (2012). The network origins of aggregate fluctuations. Econometrica, 80(5), 1977-2016.

Ahuja, R. K. (2017). Network flows: theory, algorithms, and applications. 1st Edition. Pearson. London.

Alatriste Contreras, M. G. (2014). Essais sur les propriétés structurelles et la dynamique du système économique. Thèse de doctorat en Analyse et politique économiques, GREQAM- Aix-Marseille University

Blöchl, F., Theis, F. J., Vega-Redondo, F., and Fisher, E. O. N. (2011). Vertex centralities in input-output networks reveal the structure of modern economies. Physical Review E, 83(4), 046127.

Bonacich, P. (2007). Some unique properties of eigenvector centrality. Social Networks, 29(4), 555-564.

Bramoullé, Y., Kranton, R., and D'Amours, M. (2014). Strategic interaction and network. American Economic Review, 104(3), 898-930.

Carvalho, V. M. (2013). A survey paper on recent developments of input-output analysis. Complexity Research Initiative for Systemic Instabilities. FP7-ICT-2011-7-288501-CRISIS.

Donner, R. V., Hernández-García, E., and Ser-Giacomi, E. (2017). Introduction to focus issue: complex network perspectives on flow systems. AIP Publishing.

Freeman, L. C. (1978). Centrality in social networks conceptual clarification. Social Networks, 1(3), 215239. 
Freixas, X., Parigi, B.M., and Rochet, J.C. (2000). Systemic risk, interbank relations, and liquidity provision by the central bank. Journal of Money, Credit and Banking, 32(3), 611-638.

Guimera, R., Danon, L., Diaz-Guilera, A., Giralt, F., and Arenas, A. (2003). Self-similar community structure in a network of human interactions. Physical Review E, 68(6), 065103.

Hakimi, S. L. (1964). Optimum locations of switching centers and the absolute centers and medians of a graph. Operations Research, 12(3), 450-459.

Helbing, D. (2013). Globally networked risks and how to respond. Nature, 497(7447), 51-59.

Hirschman, A. O. (1958). The strategy of economic development (Vol. 58). Yale University Press, New Haven.

Jackson, M. (2008). Social and Economic Networks. Princeton University Press: Princeton, NJ.

Kaya, T. (2017). Unraveling the Energy Use Network of Construction Sector in Turkey Using Structural Path Analysis. International Journal of Energy Economics and Policy, 7(1), 31-43.

König, M. D., Tessone, C. J., and Zenoue, Y. (2014). Nestedness in networks: A theoretical model and some applications. Theoretical Economics, 9(3), 695-752.

McNerney, J. (2009). Network properties of economic input-output networks. IIASA Interim Report. IIASA, Laxenburg, Austria: IR-09-003

McNerney, J., Fath, B. D., and Silverberg, G. (2013). Network structure of inter-industry flows. Physica A: Statistical Mechanics and its Applications, 392(24), 6427-6441.

Muniz, A. S. G., Raya, A. M., and Carvajal, C. R. (2008). Key sectors: A new proposal from network theory. Regional Studies, 42(7), 1013-1030.

Muñiz, A. S. G., Raya, A. M., and Carvajal, C. R. (2011). Core periphery valued models in input-output field: A scope from network theory. Papers in Regional Science, 90(1), 111-121.

Nuss, P., Chen, W.-Q., Ohno, H., and Graedel, T. E. (2016). Structural investigation of aluminum in the US economy using network analysis. Environmental Science Technology, 50(7), 4091-4101.

Oh, P. and Monge, P. (2016). Network theory and models. University of Southern California. Wiley Online Library. Retrieved from: https://onlinelibrary.wiley.com/doi/pdf/10.1002/9781118766804.wbiect246 https://doi.org/10.1002/9781118766804.wbiect246

Page, L., Brin, S., Motwani, R., and Winograd, T. (1999). The PageRank citation ranking: bringing order to the web. Retrieved from http://ilpubs.stanford.edu:8090/422/1/1999-66.pdf

Pauliuk, S., Majeau-Bettez, G., and Müller, D. B. (2015). A general system structure and accounting framework for socioeconomic metabolism. Journal of Industrial Ecology, 19(5), 728-741.

Pinski, G., and Narin, F. (1976). Citation influence for journal aggregates of scientific publications: Theory, with application to the literature of physics. Information Processing and Management, $12(5), 297-312$.

Prell, C. (2016). Wealth and pollution inequalities of global trade: A network and input-output approach. The Social Science Journal, 53(1), 111-121.

Prell, C., Sun, L., Feng, K., and Myroniuk, T. W. (2015). Inequalities in global trade: A cross-country comparison of trade network position, economic wealth, pollution and mortality. PloS one, 10(12), e0144453.

Proctor, C. H., and Loomis, C. P. (1951). Analysis of sociometric data. Research Methods in Social Relations, 2, 561-585.

Sabidussi, G. (1966). The centrality index of a graph. Psychometrika, 31(4), 581-603.

Shaw, M. E. (1954). Group structure and the behavior of individuals in small groups. The Journal of Psychology, 38(1), 139-149.

Smoot, M. E., Ono, K., Ruscheinski, J., Wang, P. L., and Ideker, T. (2010). Cytoscape 2.8: new features for data integration and network visualization. Bioinformatics, 27(3), 431-432.

Strogatz, S. H. (2001). Exploring complex networks. Nature, 410(6825), 268-276.

Suh, S. (2005). Theory of materials and energy flow analysis in ecology and economics. Ecological Modelling, 189(3), 251-269.

Suh, S. (2009). Handbook of input-output economics in industrial ecology (Vol. 23): Springer Science and Business Media.

Timmer, M. P., Dietzenbacher, E., Los, B., Stehrer, R., and Vries, G. J. (2015). An illustrated user guide to the world input-output database: the case of global automotive production. Review of International Economics, 23(3), 575-605.

UNSTATS (2007). Handbook of National Accounting - Input-Output Table Compilation and Analysis. United Nations

Wasserman, S. (1994). Social network analysis: Methods and applications (Vol. 8): Cambridge University press.

Wiedmann, T. O., Schandl, H., Lenzen, M., Moran, D., Suh, S., West, J., and Kanemoto, K. (2015). The material footprint of nations. Proceedings of the National Academy of Sciences, 112(20), 6271-6276. 
506 REMEF (The Mexican Journal of Economics and Finance) 